\title{
Opinion \\ Posterior urethral valves- over 5 decades in Kerala
}

\author{
Volume 5 Issue 7 - 2016 \\ Thekkanath Joseph \\ Baby Memorial Hospital, India \\ Correspondence: Thekkanath Joseph, Consultant Pediatric \\ Surgeon, Baby Memorial Hospital, India, \\ Email jorosamma@yahoo.com \\ Received: June 10,2016 | Published: November 30, 2016
}

I will share with you my experience with Posterior Urethral Valves (PUV) during the last 5 decades. In the sixties, PUV was managed with clinical diagnosis and minimal imaging studies; Very few survived, and many died with no diagnosis made. In the seventies, introduction of Voiding Cysto-urethrogram (VCU) and later Ultrasongram, (USG), Renogram and Magnetic Resonance Urogram (MRU) made dramatic changes in diagnosis and management of PUV; availability of fibre optic miniature cystoscopes helped the management.

In the seventies, eighties and early nineties, many presented with urinary symptoms, such as difficulty in voiding, crying on voiding, weak or interrupted stream, urinary tract infection (UTI), failure to thrive etc; now many will be detected by Ante Natal USG, and confirmed by Post Natal imaging modalities; some will present later with urinary symptoms; some are still missed during childhood and detected during adolescence or even later.

In late seventies, our diagnosis of PUV was still not frequent; however, by mid eighties, in Calicut Medical College, about 80 to 85 cases were diagnosed each year; this became possible due to awareness of Pediatric Physicians suspecting more cases and referring to Pediatric Surgeon for management. High index of suspicion and use of VCU more often led to frequent diagnosis. During eighties and even early nineties, only large size scope was available for children and newborn urethra did not admit the large scope.

In suspected cases, USG, VCU, Renogram, and rarely MRU and IVU will help diagnosis. Urinalysis, urea and creatinine help in treating and monitoring these children.VCU is ideally done by Pediatric Radiologist; in the absence of a dedicated pediatric Radiologist, pediatric Surgeon is next best person to do VCU; we need to get clear view of entire urethra in an oblique view; also, introduction of infection into urinary tract is to be avoided in these babies; VCU will show trabeculated urinary bladder, dilated posterior urethra, and any Vesico-Ureteric Reflux.

Management of these babies depend on the presence of UTI and also functional status; In the absence of UTI, they can undergo valve ablation using cystoscope. They need urinary drainage for a few days,

along with supportive measures and anticholinergics. Many children will need only valve ablation and regular follow up. Some of these babies may need urinary diversion, in the form of Vesicostomy, ureterostomy or pyelostomy; some children may need secondary operations like ureteric re-implantation, pyeloplasty or even unilateral nephrectomy in non- functioning kidney. Anticholinergics on long term with timed voiding have helped bladder function. All these patients need lifelong follow up with urinalysis, USG and renogram as needed; about 75 to $80 \%$ of PUV patients will be able to lead a normal life with no end stage renal disease developing; all babies with a nadir creatinine less than $0.8 \mathrm{mg}$ are likely to do well in long term with good renal function; with prompt diagnosis and management and regular follow up, most of these children can lead a normal life. Babies with nadir creatinine of more than $1.2 \mathrm{mg}$ have a high chance of going for end stage renal disease. In our country, we still need to detect PUV in many children at an early stage and treat. Any boy with UTI, hydronephrosis or VUR is likely to be associated with PUV, and we have to investigate all such children.

\section{Acknowledgements}

None.

\section{Conflicts of interest}

The authors declare no conflict of interest.

\section{Funding}

None. 